Article

\title{
Exploring the Tension between Current Cosmic Microwave Background and Cosmic Shear Data
}

\author{
Eleonora Di Valentino and Sarah Bridle * \\ Jodrell Bank Center for Astrophysics, School of Physics and Astronomy, University of Manchester, Oxford Road, \\ Manchester M13 9PL, UK; eleonora.divalentino@manchester.ac.uk \\ * Correspondence: sarah@sarahbridle.net
}

Received: 3 October 2018; Accepted: 23 October 2018; Published: 2 November 2018

check for

updates

\begin{abstract}
This paper provides a snapshot of the formal $S_{8} \equiv \sigma_{8} \sqrt{\Omega_{m} / 0.3}$ tension between Planck 2015 and the Kilo Degree Survey of $450 \mathrm{deg}^{2}$ of imaging data (KiDS-450) or the Canada France Hawaii Lensing Survey (CFHTLenS). We find that the Cosmic Microwave Bckground (CMB) and cosmic shear datasets are in tension in the standard $\Lambda$ Cold Dark Matter $(\Lambda \mathrm{CDM})$ model, and that adding massive neutrinos does not relieve the tension. If we include an additional scaling parameter on the CMB lensing amplitude $A_{\text {lens }}$, we find that this can put in agreement the Planck 2015 with the cosmic shear data. $A_{\text {lens }}$ is a phenomenological parameter that is found to be more than $2 \sigma$ higher than the expected value in the Planck 2015 data, suggesting an higher amount of lensing in the power spectra, not supported by the trispectrum analysis.
\end{abstract}

Keywords: Cosmic Microwave Bckground (CMB); S8 tension; cosmological parameters

\section{Introduction}

There is a long history of potential tension between low redshift probes of mass clustering and the Cosmic Microwave Background (CMB), and ongoing speculation that this may be evidence for new physics. Cosmology is at an exciting time. The best CMB cosmology experiment for a few years, Planck, has just released its final conclusions [1,2] [hereafter Planck 2018]. Meanwhile, some of the first constraints from the new generation of cosmic shear experiments starting to come out, fromt he Kilo Degree Survey of $450 \mathrm{deg}^{2}$ of imaging data (KiDS-450) [3-5] and the Dark Energy Survey (DES) [6,7], as well as existing results from the Canada France Hawaii Lensing Survey (CFHTLenS) [8-10]. The newest likelihoods from these surveys have not yet been released; so, in anticipation, we take stock of the current formal constraints using the current best public likelihoods from each of the Planck and KiDS collaborations, as well as the version of CFHTLenS, currently the default in the most popular cosmology code CosmoMC.

The Planck 2018 results appear to have corroborated the expectations of the standard $\Lambda$ CDM cosmological model. Despite the impressive agreement, some hints for deviations from the standard scenario have been confirmed with this release-for example, some internal inconsistencies like the tension between the constraints obtained by considering the high and low multipoles of Planck, already present in the previous data release $[2,11,12]$, or the hints at more than two standard deviations for new physics, like the $A_{\text {lens }}$ anomaly, i.e., the amplitude of the gravitational lensing in the angular power spectra different from one, or the curvature of the Universe different from zero, or modified gravity (MG). Moreover, there are the tensions between Planck and some other cosmological probes, like the direct measurements of the Hubble constant [13-15] at more than $3 \sigma$, and the tension in the $\sigma_{8}-\Omega_{m}$ plane with cosmic shear experiments at more than $2 \sigma[3-5,8-10]$.

In the literature, several solutions (see for example [16-24]) have been proposed for solving the tension in the $\sigma_{8}-\Omega_{m}$ plane. In this paper, we will revisit it using different extended cosmological 
models. We will consider the cosmic shear datasets both using the original angular scales and using conservative cuts, and we use the current latest publicly available likelihood from Planck [25], which we refer to as Planck 2015 throughout.

The paper is organized in the following way: in Section 2, we will present the codes, the method and the datasets we will use for the analysis; in Section 3, we will show our results in different cosmological scenarios, and, finally, in Section 4, we will present our conclusions.

\section{Method}

We analyze the cosmological data by considering the six cosmological parameters of the Standard $\Lambda$ CDM model, namely the baryon energy density $\Omega_{b} h^{2}$, the cold dark matter energy density $\Omega_{c} h^{2}$, the ratio between the sound horizon and the angular diameter distance at decoupling $\theta_{S}$, the reionization optical depth $\tau$, the spectral index of the scalar perturbations $n_{\mathrm{S}}$ and the amplitude of the primordial power spectrum $A_{\mathrm{S}}$.

In a second step, we add one more parameter at a time, i.e., the total neutrino mass $\Sigma m_{v}$, and the lensing amplitude $A_{\text {lens }}$ in order to find a way to relieve the $S_{8}$ tension.

All the parameters of our analysis are explored within the range of the conservative flat priors reported in Table 1.

Table 1. Flat priors on the cosmological parameters assumed in this work.

\begin{tabular}{cc}
\hline Parameter & Prior \\
\hline$\Omega_{b} h^{2}$ & {$[0.013,0.033]$} \\
$\Omega_{\mathrm{c}} h^{2}$ & {$[0.001,0.99]$} \\
$\theta_{S}$ & {$[0.5,10]$} \\
$\tau$ & {$[0.01,0.8]$} \\
$n_{\mathrm{S}}$ & {$[0.7,1.3]$} \\
$\log A$ & {$[1.7,5.0]$} \\
\hline$\Sigma m_{v}$ & {$[0,5]$} \\
\hline$A_{\text {lens }}$ & {$[0,10]$} \\
\hline
\end{tabular}

We constrain the parameters by analyzing the full range of the 2015 Planck temperature power spectrum $(2 \leq \ell \leq 2500)$ together with the low multipole polarization data $(2 \leq \ell \leq 29)$ [25], and we call this combination "Planck TT". To understand the impact of the polarization data on our results, we repeat the analysis by including the Planck high multipoles polarization data [25], and we refer to this combination as "Planck TTTEEE". At the time of writing, only the Planck 2015 data is publicly available, even though preprint papers of the final data have been released. Therefore, in this analysis, we use the Planck 2015 data. However, given the similarities between the 2015 and the final Planck constraints, we do not expect the conclusions of this paper to change qualitatively with the new likelihood.

We compare these constraints with those obtained with the cosmic shear data from the Canada France Hawaii Lensing Survey (CFHTLenS) [8,9], with the original cut, that we call 'CFHTLenS', and with the conservative cut as described in [23], which we call 'CFHTLenS-linear-cut' (The default WL.ini in CosmoMC is CFHTLenS-linear-cut, i.e., CFHTLENS_6bin_ultra_conservative.dataset, while the original cut CFHTLenS is CFHTLENS_6bin.dataset). The CFHTLenS in CosmoMC does not include the baryonic feedback and the photo-z errors, while the CFHTLenS-linear-cut to the data is free from much of the systematics. For the latest measurements, see the discussion in Ref. [10] and the release at [26]. Moreover, we consider the Kilo Degree Survey of $450 \mathrm{deg}^{2}$ of imaging data (KiDS-450) [3-5], also in this case with the original cut, which we call 'KiDS', and with the conservative cuts as described in [27], which we call 'KiDS-linear-cut'. We note that the KiDS data has recently been re-analysed by Ref. [28], who modify the covariance matrix and bin positions to correct approximations made in the original release. These changes reduce the tension with Planck by increasing the clustering 
amplitude measured by cosmic shear by up to half a sigma. In the absence of an official update to the KiDS data, we have chosen to stick with the publicly available version provided by the KiDS team.

Finally, we consider a combination of data including a Gaussian prior on $H_{0}$ (i.e., $H_{0}=73.24 \pm 1.75 \mathrm{~km} \mathrm{~s}^{-1} \mathrm{Mpc}^{-1}$ [14]), referred as "R16"; the baryon acoustic oscillation data from 6dFGS [29], SDSS-MGS [30], BOSSLOWZ [31] and CMASS-DR11 [31] surveys as was done in [32], called "BAO"; and the Joint light-curve analysis (JLA) sample of Supernovae Type Ia comprising 740 luminosity distance measurements in the redshift interval $z \in[0.01,1.30]$ [33], referred as "JLA".

In order to analyze these datasets statistically exploring the different cosmological scenarios, we have used the publicly available Monte-Carlo Markov Chain package cosmomc [34], with a convergence diagnostic based on the Gelman and Rubin statistic. It implements an efficient sampling of the posterior distribution using the fast/slow parameter decorrelations [35], including also the support for the Planck data release 2015 Likelihood Code [25] (see [36]). Finally, we make use of the modified cosmomc modules and associated data files for weak lensing tomography cosmology fitting with KiDS [3,37] (see [38]).

\section{Results}

\subsection{Standard Cosmological Model}

In Table A1 and in Figure 1, we show the constraints for $\sigma_{8}$ and $\Omega_{m}$ for Planck and the cosmic shear experiments considered in this work, i.e., KiDS and CFHTLenS, for the original and the conservative scale cut, assuming the standard $\Lambda \mathrm{CDM}$ model. The conservative cut is used to mitigate the uncertainty over the nonlinear modeling scheme. Moreover, we compute the combination $S_{8} \equiv \sigma_{8} \sqrt{\Omega_{m} / 0.3}$, reported in Figure 2, in order to quantify the tension between the datasets in the 2D dimensions, and we compare the full combination of datasets KiDS + BAO + JLA + R16 with Planck. In fact, if we compare the constraints on $\sigma_{8}$ and $\Omega_{m}$, reported in Table A1, from the Planck satellite with KiDS or CFHTLenS, they seem perfectly in agreement. However, we can see from the Figures 1 and 2 that Planck TTTEEE is in disagreement with both CFHTLenS and KiDS, in agreement with each other, at more than $3 \sigma$ and $2 \sigma$, respectively, while the tension disappears if we consider the conservative cuts for both the experiments. However, when combining KiDS with all the other cosmological probes considered here, namely BAO + JLA + R16, also for the conservative cut, the tension with Planck appears again at more than $2 \sigma$. The tension T in unit of $\sigma$ can be quantified looking at the $S_{8}$ constraints in Figure 2 and Table A1 and computing:

$$
T=\frac{S_{8, i}-S_{8, j}}{\sqrt{\sigma_{S_{8, i}}^{2}+\sigma_{S_{8, j}}^{2}}} .
$$

While this tension between Planck and the cosmic shear experiments can be due to the presence of systematics in one or all the experiments, it is interesting to investigate if, by changing the assumed cosmological model, this tension can be mitigated or solved completely because the constraints obtained are always model dependent. 

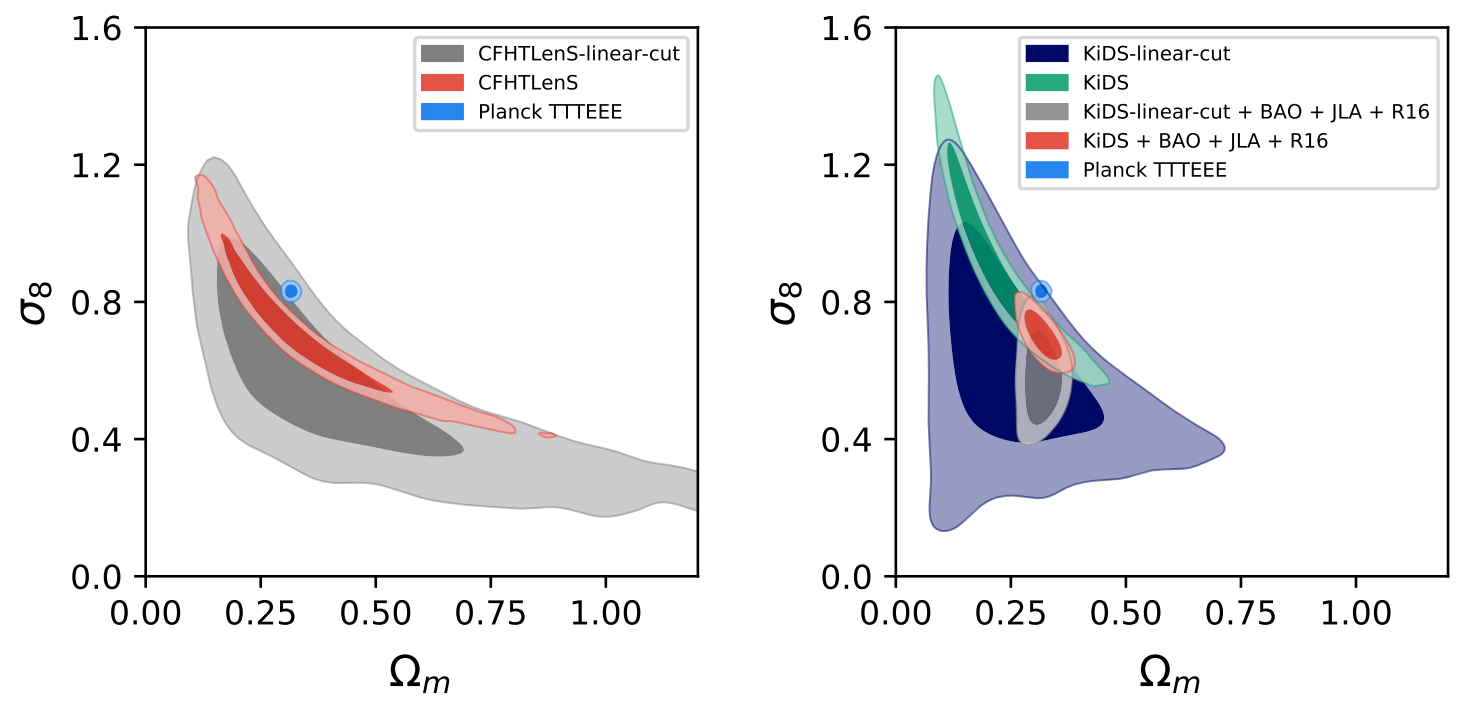

Figure 1. Constraints at $68 \%$ and $95 \%$ confidence levels on the $\sigma_{8}$ vs. $\Omega_{m}$ plane for several combination of datasets in the $\Lambda$ CDM scenario. In both the cases, Planck is in tension at more than $2 \sigma$ with the cosmic shear experiments with the original cut, while is in agreement considering their conservative cut.

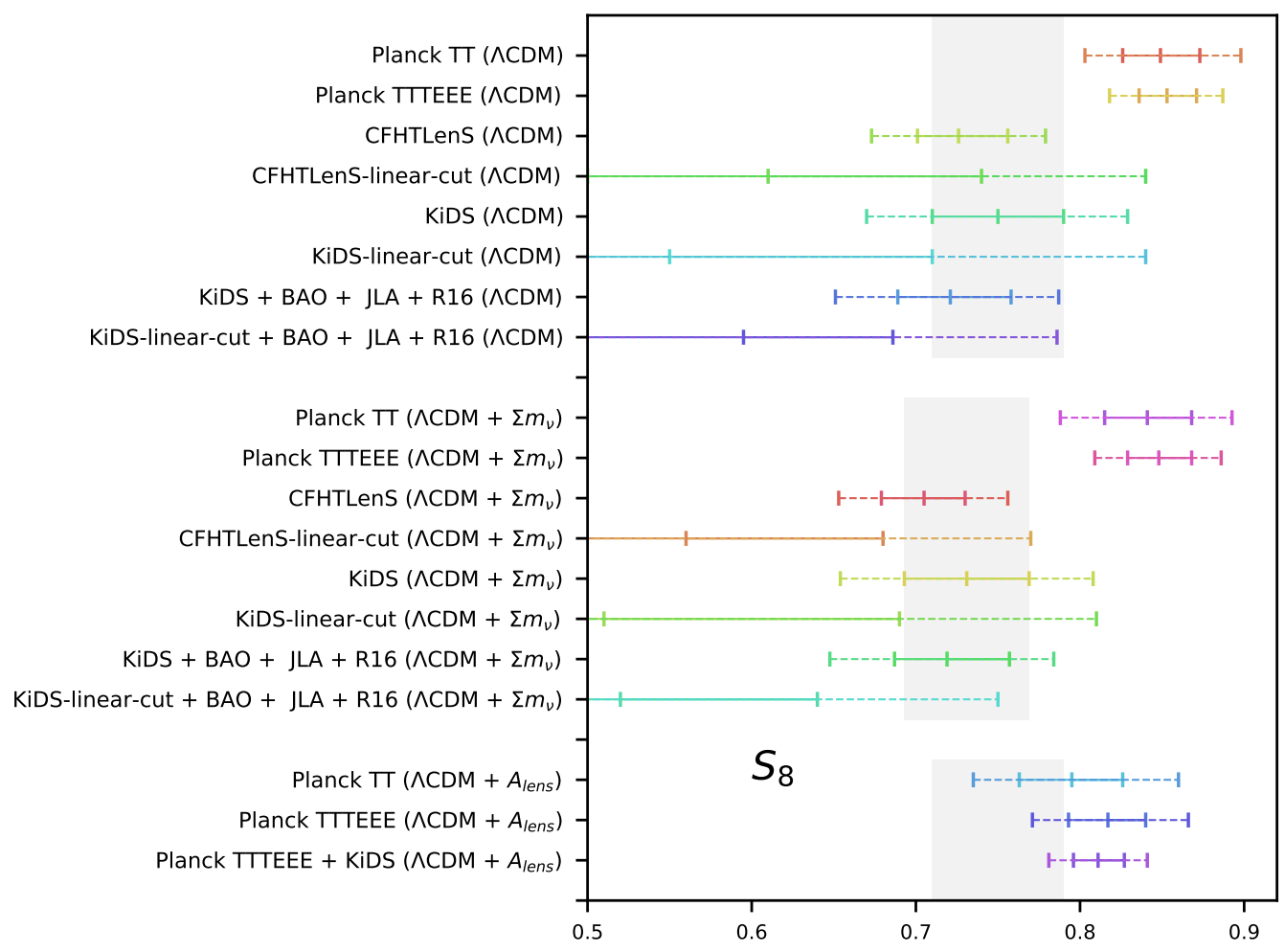

Figure 2. Constraints at $68 \%$ (solid) and $95 \%$ (dashed) on $S_{8}$ for several combination of datasets and models considered in this work. The gray band corresponds to the KiDS bounds in each cosmological scenario.

\subsection{Massive Neutrinos}

In Table A2 and in Figure 3, we show the constraints for $\sigma_{8}, \Omega_{m}$ and $S_{8}$ for Planck, KiDS and CFHTLenS, for both the original and the conservative scale cuts, assuming the $\Lambda \mathrm{CDM}+\Sigma m_{v}$ model.

Also in this case, if we compare the constraints on $\sigma_{8}$ and $\Omega_{m}$, reported in Table A2, from the Planck satellite with KiDS or CFHTLenSt, they seem perfectly in agreement. Usually, massive neutrinos 
are included in the analysis to try to solve the tension between Planck and the cosmic shear experiments because, when introduced, the $\sigma_{8}$ bound shifts towards lower values [22,39-42]. However, we can see from the Figure 3 that Planck TTTEEE is in disagreement with both CFHTLenS and KiDS even when varying massive neutrinos, at more than $3 \sigma$ and $2 \sigma$ respectively. Also in this case, the tension is reduced if we consider the conservative cuts for both the experiments. Moreover, also in this case, when considering conservative KiDS + BAO + JLA + R16, the tension with Planck is restored at more than $2 \sigma$, as we can see looking at the $S_{8}$ constraints in Figure 2 and Table A2.
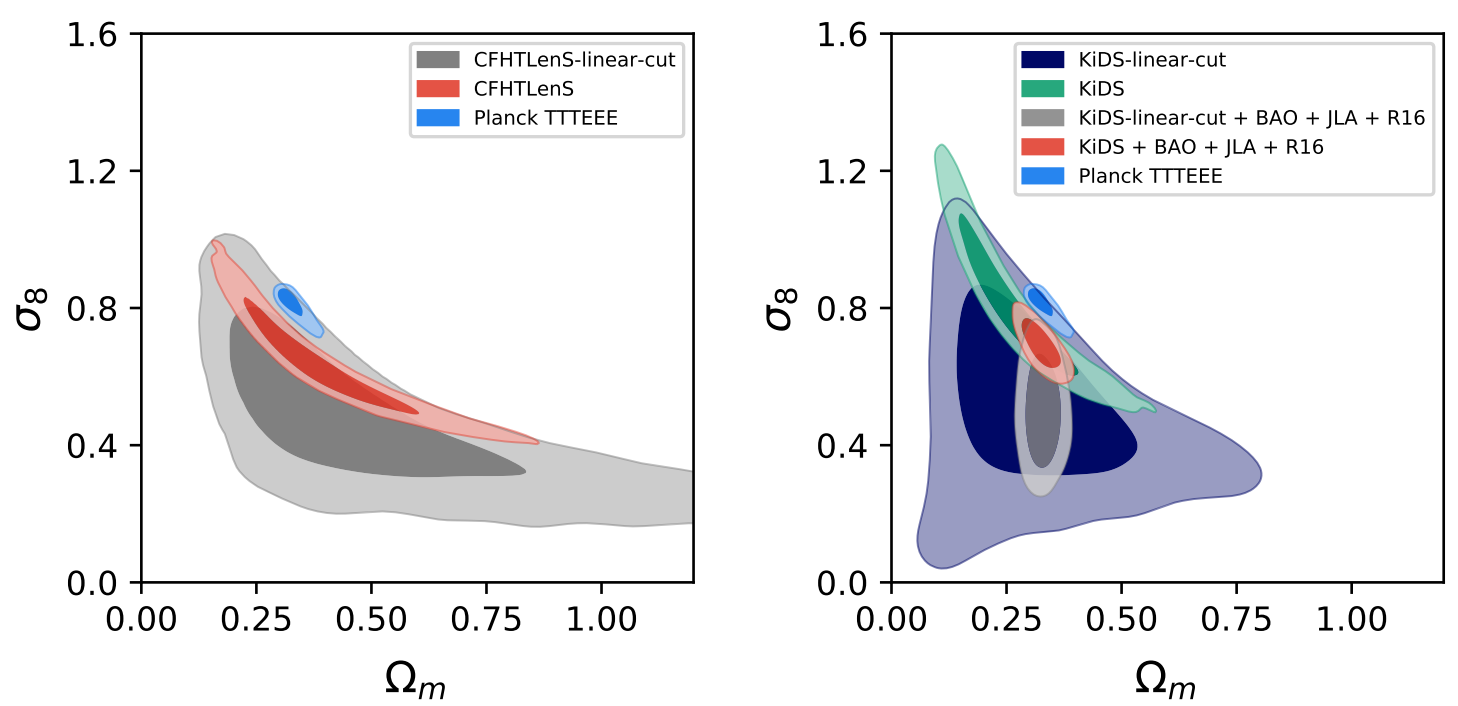

Figure 3. Constraints at $68 \%$ and $95 \%$ confidence levels on the $\sigma_{8}$ vs. $\Omega_{m}$ plane for several combination of datasets in the $\Lambda \mathrm{CDM}+\Sigma m_{v}$ model. In both of the cases, Planck is still in tension at more than $2 \sigma$ with the cosmic shear experiments with the original cut, while is in agreement considering their conservative cut.

\subsection{The Lensing Amplitude}

In Table A3 and in Figure 4, we show the constraints for $\sigma_{8}, \Omega_{m}$ and $S_{8}$ for Planck assuming the $\Lambda \mathrm{CDM}+A_{\text {lens }}$ model. In this case, we compare the results with the constraints obtained for the parameters listed before for the cosmic shear experiments in the $\Lambda \mathrm{CDM}$ case because the $A_{\text {lens }}$ parameter is just an effective parameter, with no physical meaning, that simply rescales the the lensing amplitude in the CMB spectra, and it is used for testing theoretical assumptions and systematics. See Ref. [43]. We consider here this $A_{\text {lens }}$ parameter because this extension of the $\Lambda C D M$ model is different from the expected value at more than $2 \sigma$, for the Planck dataset used in this work [32], and has been very recently confirmed with more statistical significance in the new Planck release [2]. This indication for a wrong amount of lensing in the $\mathrm{CMB}$ power spectra is not confirmed by the lensing reconstruction data, i.e., the trispectrum analysis, and is very robust (see Ref. [41,44,45]). Future CMB data will help in understanding this parameter, as shown in Ref. [46], while, in Refs. [2,24], it has been shown that a possible explanation for the $A_{\text {lens }}$ parameter is the degeneracy with the MG parameters. Another possibility is that $A_{\text {lens }}$ takes into account unresolved systematics. Further possible theoretical explanations have been analyzed in Refs. [47-50]. Moreover, varying $A_{\text {lens }}$ is considered in the literature a possible conservative way of using the Planck data marginalizing over the systematics in the data because an incorrect amount of lensing can bias some correlated parameters like massive neutrinos [22,41,44,45,51]. In addition, when $A_{\text {lens }}$ is varying, it is possible to solve the tension between the constraints coming from the high and low multipoles of Planck $[2,11,12]$.

A consequence of varying $A_{\text {lens }}$ in our work is that Planck TTTEEE is now perfectly in agreement with KiDS or CFHTLenS, in both the scale cuts, as we can see in Figure 4 in the $\sigma_{8}-\Omega_{m}$ plane, or by looking at the $S_{8}$ constraints in Figure 2. Also in this case, the agreement improves in a significant way 
if we consider the conservative cuts for both the experiments, but, looking at the plots, is still within the $68 \%$ c.l. also with the original cut and when considering KiDS + BAO + JLA + R16.
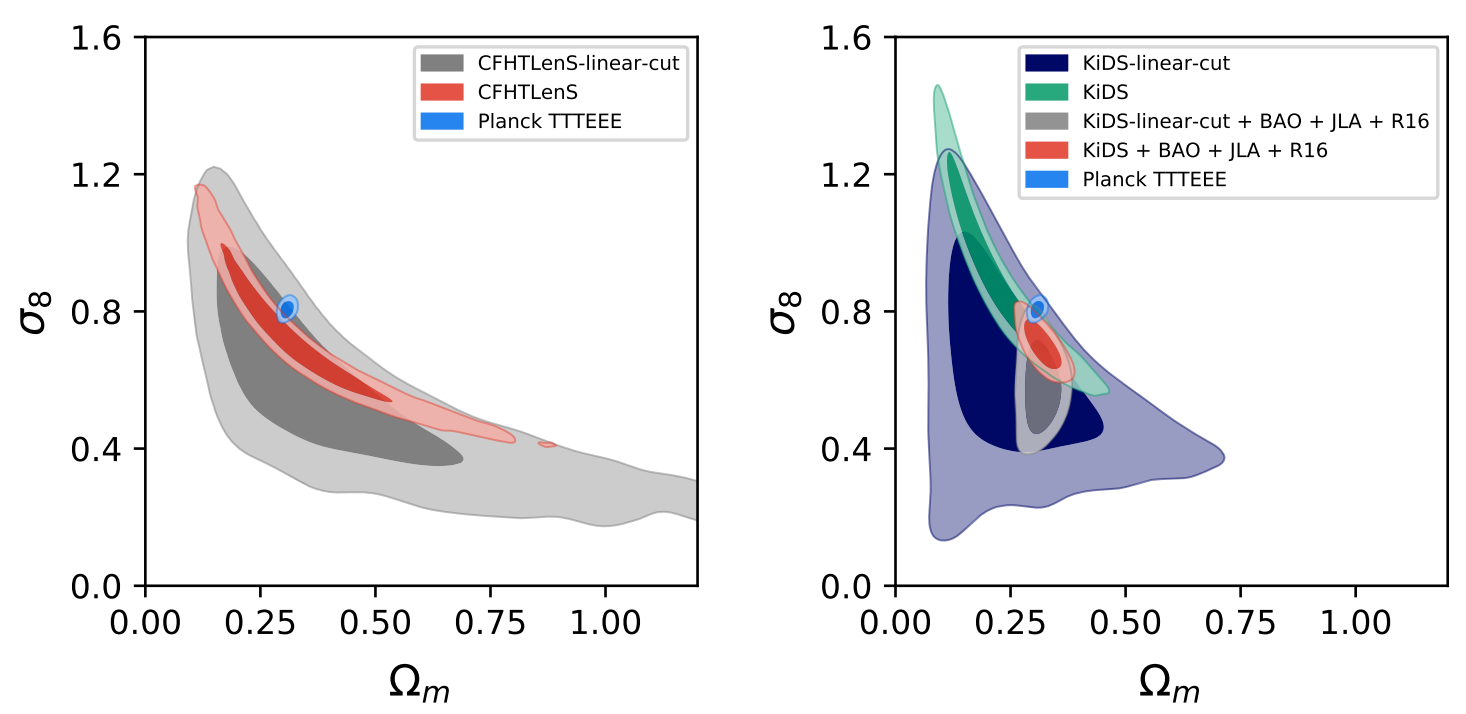

Figure 4. Constraints at $68 \%$ and $95 \%$ confidence levels on the $\sigma_{8}$ vs. $\Omega_{m}$ plane for several combination of datasets in the $\Lambda \mathrm{CDM}+A_{\text {lens }}$ model. In both of the plots, Planck shifts in agreement with the cosmic shear experiments within $2 \sigma$.

\section{Conclusions}

We have investigated the matter fluctuation amplitude $\left(S_{8}\right)$ tension between the latest publicly available cosmic shear experiment (KiDS) likelihood and the latest publicly available CMB dataset in the standard cosmological model $\Lambda \mathrm{CDM}$. We have also shown results using the most commonly used CFHTLenS dataset. We show results for both the original and the conservative (linear theory only) cut for both cosmic shear experiments. We find that there is a tension using original (nonlinear) scales for cosmic shear, but this disappears on using the conservative cut.

We considered extensions to $\Lambda \mathrm{CDM}$ to try to resolve the tension. We find that massive neutrinos do not relieve the tension because they change the $\sigma_{8}$ constraint in the right direction, simultaneously with the $\Omega_{m}$ value in the wrong one, as discussed in [41]. However, allowing a rescaling of the CMB lensing amplitude $A_{\text {lens }}$ does resolve the tension. $A_{\text {lens }}$ is a phenomenological parameter that is found to be more than $2 \sigma$ higher than the expected value in the Planck 2015 data, suggesting an higher amount of lensing in the power spectra, not supported by the trispectrum analysis. We found that this incorrect amount of lensing can be responsible for the tension between the Planck and the cosmic shear data.

We already discuss the possibility of having systematics in Planck, which could be described by the $A_{\text {lens }}$ parameter that we considered in this analysis. There are also known systematics in the weak lensing experiments we considered, which could change our conclusions. For example, see the long list in Ref. [52] for CFHTLenS, where however the authors show that the approximations used in the analysis have a negligible impact on the cosmological parameter constraints, or see the re-analysis of the KiDS data done by Ref. [28], which improves the agreement with Planck of half a sigma. We await with interest the new likelihoods from Planck 2018 and the Dark Energy Survey [6,7] to update our work. 
Author Contributions: Conceptualization, E.D.V.; methodology, E.D.V.; formal analysis, E.D.V.; writing—original draft preparation, E.D.V.; writing - review and editing, S.B.; funding acquisition, S.B.

Funding: This research was funded by the European Research Council in the form of a Consolidator Grant No. 681431.

Acknowledgments: We would like to thank Catherine Heymans and Shahab Joudaki for stimulating discussions and suggestions. We thank the CFHTLenS, KiDS and Planck teams for making their data available to the community. E.D.V. and S.B. acknowledge support from the European Research Council in the form of a Consolidator Grant No. 681431.

Conflicts of Interest: The authors declare no conflict of interest.

\section{Appendix A}

In this appendix, we show the constraints on cosmological parameters from the different analyses performed in this paper.

Table A1. $68 \%$ credible intervals for cosmological parameters for several data combination in the $\Lambda \mathrm{CDM}$ model.

\begin{tabular}{|c|c|c|c|c|c|c|c|}
\hline Parameter & Planck TT & CFHTLenS-linear-cut & it CFHTLenS & KiDS-linear-cut & KiDS & $\begin{array}{l}\text { Planck TT + } \\
\text { KiDS }\end{array}$ & $\begin{array}{c}\text { Planck TT + } \\
\text { KiDS-linear-cut }\end{array}$ \\
\hline$\Omega_{\mathrm{m}}$ & $0.315 \pm 0.013$ & $0.35 \pm 0.16$ & $0.44 \pm 0.33$ & $0.222 \pm 0.088$ & $0.26 \pm 0.12$ & $0.2911 \pm 0.0083$ & $0.299 \pm 0.010$ \\
\hline$\sigma_{8}$ & $0.830 \pm 0.015$ & $0.72 \pm 0.16$ & $0.58 \pm 0.21$ & $0.92 \pm 0.20$ & $0.63 \pm 0.21$ & $0.8221 \pm 0.0062$ & $0.8279 \pm 0.0072$ \\
\hline$S_{8}$ & $\begin{array}{c}\text { Planck } \\
\text { TTTEEE }\end{array}$ & $\begin{array}{c}\text { Planck TTTEEE }+ \text { P } \\
\text { KiDS }\end{array}$ & $\begin{array}{l}\text { Planck TTTEEE + } \\
\text { KiDS-linear-cut }\end{array}$ & $\begin{array}{l}\text { CFHTLenS + } \\
\text { KiDS }\end{array}$ & $\begin{array}{l}\text { CFHTLenS-linear-cut } \\
\text { + KiDS-linear-cut }\end{array}$ & $\begin{array}{c}\text { KiDS + BAO + } \\
\text { JLA + R16 }\end{array}$ & $\begin{array}{l}\text { KiDS-linear-cut + } \\
\text { BAO + JLA + R16 }\end{array}$ \\
\hline$\Omega_{\mathrm{m}}$ & $0.3162 \pm 0.0090$ & $0.3006 \pm 0.0065$ & $0.3077 \pm 0.0078$ & $0.245 \pm 0.085$ & $0.28 \pm 0.11$ & $0.319 \pm 0.027$ & $0.316 \pm 0.026$ \\
\hline
\end{tabular}

Table A2. 68\% credible intervals for cosmological parameters for several data combination in the $\Lambda \mathrm{CDM}+\Sigma m_{v}$ model.

\begin{tabular}{|c|c|c|c|c|c|c|c|}
\hline Parameter & r Planck TT & CFHTLenS & CFHTLenS-linear-cut & KiDS & KiDS-linear-cut & $\begin{array}{l}\text { Planck TT + } \\
\text { KiDS }\end{array}$ & $\begin{array}{c}\text { Planck TT + } \\
\text { KiDS-linear-cut }\end{array}$ \\
\hline$\Omega_{\mathrm{m}}$ & $0.344 \pm 0.041$ & $0.41 \pm 0.16$ & $0.44 \pm 0.55$ & $0.27 \pm 0.10$ & $0.31 \pm 0.15$ & $0.306 \pm 0.027$ & $0.313 \pm 0.027$ \\
\hline$\sigma_{8}$ & $0.790 \pm 0.051$ & $0.64 \pm 0.12$ & $0.47 \pm 0.17$ & $0.81 \pm 0.17$ & $0.53 \pm 0.19$ & $0.799 \pm 0.037$ & $0.807 \pm 0.037$ \\
\hline \multirow[t]{2}{*}{$S_{8}$} & $0.841 \pm 0.026$ & $0.705 \pm 0.026$ & $0.56 \pm 0.11$ & $0.731 \pm 0.039$ & $0.51 \pm 0.16$ & $0.805 \pm 0.018$ & $0.822 \pm 0.024$ \\
\hline & Planck TTTEEE & $\begin{array}{l}\text { Planck TTTEEE } \\
+ \text { KiDS }\end{array}$ & $\begin{array}{cc}\text { Planck TTTEEE }+ & \mathrm{C} \\
\text { KiDS-linear-cut }\end{array}$ & $\begin{array}{l}\text { CFHTLenS-linear-cut } \\
+ \text { KiDS-linear-cut }\end{array}$ & $\begin{array}{l}\mathrm{KiDS}+\mathrm{BAO}+ \\
\mathrm{JLA}+\mathrm{R} 16\end{array}$ & $\begin{array}{l}\text { KiDS-linear-cut + } \\
\text { BAO + JLA + R16 }\end{array}$ & \\
\hline$\Omega_{\mathrm{m}}$ & $0.329 \pm 0.023$ & $0.315 \pm 0.024$ & $0.318 \pm 0.020$ & $0.35 \pm 0.15$ & $0.325 \pm 0.028$ & $0.328 \pm 0.026$ & \\
\hline$\sigma_{8}$ & $0.811 \pm 0.033$ & $0.807 \pm 0.036$ & $0.817 \pm 0.029$ & $0.61 \pm 0.17$ & $0.693 \pm 0.048$ & $0.50 \pm 0.11$ & \\
\hline$S_{8}$ & $0.848 \pm 0.020$ & $0.824 \pm 0.016$ & $0.840 \pm 0.017$ & $0.611 \pm 0.090$ & $0.719 \pm 0.035$ & $0.52 \pm 0.11$ & \\
\hline
\end{tabular}

Table A3. $68 \%$ credible intervals for cosmological parameters for several data combination in the $\Lambda \mathrm{CDM}+A_{\text {lens }}$ model.

\begin{tabular}{cccccc}
\hline Parameter & Planck TT & $\begin{array}{c}\text { Planck TT + } \\
\text { KiDS-linear-cut }\end{array}$ & Planck TTTEEE & $\begin{array}{c}\text { Planck TTTEEE + } \\
\text { KiDS }\end{array}$ & $\begin{array}{c}\text { Planck TTTEEE + } \\
\text { KiDS-linear-cut }\end{array}$ \\
\hline$\Omega_{\mathrm{m}}$ & $0.295 \pm 0.015$ & $0.278 \pm 0.012$ & $0.329 \pm 0.023$ & $0.2919 \pm 0.0074$ & $0.2983 \pm 0.0086$ \\
$\sigma_{8}$ & $0.802 \pm 0.018$ & $0.8138 \pm 0.0094$ & $0.806 \pm 0.017$ & $0.8224 \pm 0.0056$ & $0.8271 \pm 0.0063$ \\
$S_{8}$ & $0.795 \pm 0.032$ & $0.783 \pm 0.026$ & $0.817 \pm 0.024$ & $0.811 \pm 0.015$ & $0.825 \pm 0.018$ \\
\hline
\end{tabular}

\section{References}

1. Akrami, Y.; Arroja, F.; Ashdown, M.; Aumont, J.; Baccigalupi, C.; Ballardini, M.; Banday, A.J.; Barreiro, R.B.; Bartolo, N.; Basak, S.; et al. Planck 2018 results. I. Overview and the cosmological legacy of Planck. arXiv 2018, arXiv:1807.06205.

2. Aghanim, N.; Akrami, Y.; Ashdown, M.; Aumont, J.; Baccigalupi, C.; Ballardini, M.; Banday, A.J.; Barreiro, R.B.; Bartolo, N.; Basak, S.; et al. Planck 2018 results. VI. Cosmological parameters. arXiv 2018, arXiv:1807.06209.

3. Hildebrandt, H.; Viola, M.; Heymans, C.; Joudaki, S.; Kuijken, K.; Blake, C.; Erben, T.; Joachimi, B.; Klaes, D.; Miller, L.T.; et al. KiDS-450: Cosmological parameter constraints from tomographic weak gravitational lensing. arXiv 2016, arXiv:1606.05338. 
4. Kuijken, K.; Heymans, C.; Hildebrandt, H.; Nakajima, R.; Erben, T.; de Jong, J.T.; Viola, M.; Choi, A.; Hoekstra, H.; Miller, L.; et al. Gravitational Lensing Analysis of the Kilo Degree Survey. Mon. Not. R. Astron. Soc. 2015, 454, 3500-3532. [CrossRef]

5. Fenech Conti, I.; Herbonnet, R.; Hoekstra, H.; Merten, J.; Miller, L.; Viola, M. Calibration of weak-lensing shear in the Kilo-Degree Survey. Mon. Not. R. Astron. Soc. 2017, 467, 1627-1651.

6. Abbott, T.M.; Abdalla, F.B.; Alarcon, A.; Aleksić, J.; Allam, S.; Allen, S.; Amara, A.; Annis, J.; Asorey, J.; Avila, S.; et al. Dark energy survey year 1 results: Cosmological constraints from galaxy clustering and weak lensing. Phys. Rev. D 2018, 98, 043526. [CrossRef]

7. Troxel, M.A.; MacCrann, N.; Zuntz, J.; Eifler, T.F.; Krause, E.; Dodelson, S.; Gruen, D.; Blazek, J.; Friedrich, O.; Samuroff, S.; et al. Dark Energy Survey Year 1 results: Cosmological constraints from cosmic shear. Phys. Rev. D 2018, 98, 043528. [CrossRef]

8. Heymans, C.; Van Waerbeke, L.; Miller, L.; Erben, T.; Hildebrandt, H.; Hoekstra, H.; Kitching, T.D.; Mellier, Y.; Simon, P.; Bonnett, C.; et al. CFHTLenS: The Canada-France-Hawaii Telescope Lensing Survey. Mon. Not. R. Astron. Soc. 2012, 427, 146-166. [CrossRef]

9. Erben, T.; Hildebrandt, H.; Miller, L.; van Waerbeke, L.; Heymans, C.; Hoekstra, H.; Kitching, T.D.; Mellier, Y.; Benjamin, J.; Blake, C.; et al. CFHTLenS: The Canada-France-Hawaii Telescope Lensing Survey-Imaging Data and Catalogue Products. Mon. Not. R. Astron. Soc. 2013, 433, 2545-2563. [CrossRef]

10. Joudaki, S.; Blake, C.; Heymans, C.; Choi, A.; Harnois-Deraps, J.; Hildebrandt, H.; Joachimi, B.; Johnson, A.; Mead, A.; Parkinson, D.; et al. CFHTLenS revisited: Assessing concordance with Planck including astrophysical systematics. Mon. Not. R. Astron. Soc. 2013, 465, stw2665. [CrossRef]

11. Aghanim, N.; Akrami, Y.; Ashdown, M.; Aumont, J.; Baccigalupi, C.; Ballardini, M.; Banday, A.J.; Barreiro, R.B.; Bartolo, N.; Basak, S.; et al. Planck intermediate results-LI. Features in the cosmic microwave background temperature power spectrum and shifts in cosmological parameters. Astron. Astrophys. 2017, 607, A95. [CrossRef]

12. Addison, G.E.; Huang, Y.; Watts, D.J.; Bennett, C.L.; Halpern, M.; Hinshaw, G.; Weiland, J.L. Quantifying discordance in the 2015 Planck CMB spectrum. Astrophys. J. 2016, 818, 312. [CrossRef]

13. Riess, A.G.; Macri, L.; Casertano, S.; Lampeitl, H.; Ferguson, H.C.; Filippenko, A.V.; Jha, S.W.; Li, W.; Chornock, R. A 3\% solution: determination of the Hubble constant with the Hubble Space Telescope and Wide Field Camera 3. Astrophys. J. 2011, 732, 129. [CrossRef]

14. Riess, A.G.; Macri, L.M.; Hoffmann, S.L.; Scolnic, D.; Casertano, S.; Filippenko, A.V.; Tucker, B.E.; Reid, M.J.; Jones, D.O.; Silverman, J.M.; et al. A 2.4\% determination of the local value of the Hubble constant. Astrophys. J. 2016, 826, 56. [CrossRef]

15. Riess, A.G.; Casertano, S.; Yuan, W.; Macri, L.; Bucciarelli, B.; Lattanzi, M.G.; MacKenty, J.W.; Bowers, J.B.; Zheng, W.; Filippenko, A.V.; et al. Milky Way Cepheid Standards for Measuring Cosmic Distances and Application to Gaia DR2: Implications for the Hubble Constant. Astrophys. J. 2018, 861, 126. [CrossRef]

16. Kunz, M.; Nesseris, S.; Sawicki, I. Using dark energy to suppress power at small scales. Phys. Rev. D 2015, 92, 063006. [CrossRef]

17. Enqvist, K.; Nadathur, S.; Sekiguchi, T.; Takahashi, T. Decaying dark matter and the tension in $\sigma_{8} . J$. Cosmol. Astropart. Phys. 2015, 9, 067. [CrossRef]

18. Hlozek, R.; Grin, D.; Marsh, D.J.; Ferreira, P.G. A search for ultralight axions using precision cosmological data. Phys. Rev. D 2015, 91, 103512. [CrossRef]

19. Ichiki, K.; Yoo, C.M.; Oguri, M. Relationship between the CMB, SZ Cluster Counts, and Local Hubble Parameter Measurements in a Simple Void Model. Phys. Rev. D 2016, 93, 023529. [CrossRef]

20. Dossett, J.N.; Ishak, M.; Parkinson, D.; Davis, T.M. Constraints and tensions in testing general relativity from Planck and CFHTLenS data including intrinsic alignment systematics. Phys. Rev. D 2015, 92, 023003. [CrossRef]

21. Gomez-Valent, A.; Sola, J. Relaxing the $\sigma_{8}$-tension through running vacuum in the Universe. Europhys. Lett. 2017, 120, 39001. [CrossRef]

22. McCarthy, I.G.; Bird, S.; Schaye, J.; Harnois-Deraps, J.; Font, A.S.; Van Waerbeke, L. The BAHAMAS project: The CMB-large-scale structure tension and the roles of massive neutrinos and galaxy formation. Mon. Not. R. Astron. Soc. 2018, 476, 2999-3030. [CrossRef] 
23. Ade, P.A.; Aghanim, N.; Arnaud, M.; Ashdown, M.; Aumont, J.; Baccigalupi, C.; Banday, A.J.; Barreiro, R.B.; Bartolo, N.; Battaner, E.; et al. Planck 2015 results. XIV. Dark energy and modified gravity. Astron. Astrophys. 2016, 594, A14. [CrossRef]

24. Di Valentino, E.; Melchiorri, A.; Silk, J. Cosmological hints of modified gravity? Phys. Rev. D 2016, 93, 023513. [CrossRef]

25. Aghanim, N.; Arnaud, M.; Ashdown, M.; Aumont, J.; Baccigalupi, C.; Banday, A.J.; Barreiro, R.B.; Bartlett, J.G.; Bartolo, N.; Battaner, E.; et al. Planck 2015 results. XI. CMB power spectra, likelihoods, and robustness of parameters. Astron. Astrophys. 2016, 594, A11. [CrossRef]

26. Available online: https://github.com/sjoudaki/cfhtlens_revisited (accessed on 15 September 2018).

27. Joudaki, S.; Blake, C.; Johnson, A.; Amon, A.; Asgari, M.; Choi, A.; Erben, T.; Glazebrook, K.; Harnois-Déraps, J.; Heymans, C.; et al. KiDS-450 + 2dFLenS: Cosmological parameter constraints from weak gravitational lensing tomography and overlapping redshift-space galaxy clustering. Mon. Not. R. Astron. Soc. 2018, 474, 4894-4924. [CrossRef]

28. Troxel, M.A.; Krause, E.; Chang, C.; Eifler, T.F.; Friedrich, O.; Gruen, D.; MacCrann, N.; Chen, A.; Davis, C.; DeRose, J.; et al. Survey geometry and the internal consistency of recent cosmic shear measurements. Mon. Not. R. Astron. Soc. 2018. [CrossRef]

29. Beutler, F.; Blake, C.; Colless, M.; Jones, D.H.; Staveley-Smith, L.; Campbell, L.; Parker, Q.; Saunders, W.; Watson, F. The 6dF Galaxy Survey: Baryon Acoustic Oscillations and the Local Hubble Constant. Mon. Not. R. Astron. Soc. 2011, 416, 3017-3032. [CrossRef]

30. Ross, A.J.; Samushia, L.; Howlett, C.; Percival, W.J.; Burden, A.; Manera, M. The Clustering of the SDSS DR7 Main Galaxy Sample I: A 4 per cent Distance Measure at $\mathrm{z}=0.15$. Mon. Not. R. Astron. Soc. 2015, 449, 835-847. [CrossRef]

31. Anderson, L.; Aubourg, É.; Bailey, S.; Beutler, F.; Bhardwaj, V.; Blanton, M.; Bolton, A.S.; Brinkmann, J.; Brownstein, J.R.; Burden, A.; et al. The clustering of galaxies in the SDSS-III Baryon Oscillation Spectroscopic Survey: Baryon acoustic oscillations in the Data Releases 10 and 11 Galaxy samples. Mon. Not. R. Astron. Soc. 2014, 441, 24-62. [CrossRef]

32. Ade, P.A.; Aghanim, N.; Arnaud, M.; Ashdown, M.; Aumont, J.; Baccigalupi, C.; Banday, A.J.; Barreiro, R.B.; Bartlett, J.G.; Bartolo, N.; et al. Planck 2015 results. XIII. Cosmological parameters. Astron. Astrophys. 2016, 594, A13.

33. Betoule, M.E.; Kessler, R.; Guy, J.; Mosher, J.; Hardin, D.; Biswas, R.; Astier, P.; El-Hage, P.; Konig, M.; Kuhlmann, S.; et al. Improved cosmological constraints from a joint analysis of the SDSS-II and SNLS supernova samples. Astron. Astrophys. 2014, 568, A22. [CrossRef]

34. Lewis, A.; Bridle, S. Cosmological parameters from CMB and other data: A Monte Carlo approach. Phys. Rev. D 2002, 66, 103511. [CrossRef]

35. Lewis, A. Efficient sampling of fast and slow cosmological parameters. Phys. Rev. D 2013, 87, 103529. [CrossRef]

36. Available online: http:/ / cosmologist.info/cosmomc/ (accessed on 15 September 2018).

37. Joudaki, S.; Mead, A.; Blake, C.; Choi, A.; de Jong, J.; Erben, T.; Fenech Conti, I.; Herbonnet, R.; Heymans, C.; Hildebrandt, H.; et al. KiDS-450: Testing extensions to the standard cosmological model. Mon. Not. R. Astron. Soc. 2017, 471, 1259. [CrossRef]

38. Available online: https:/ / github.com/sjoudaki/kids450 (accessed on 15 September 2018).

39. Giusarma, E.; Di Valentino, E.; Lattanzi, M.; Melchiorri, A.; Mena, O. Relic neutrinos, thermal axions, and cosmology in early 2014. Phys. Rev. D 2014, 90, 043507. [CrossRef]

40. MacCrann, N.; Zuntz, J.; Bridle, S.; Jain, B.; Becker, M.R. Cosmic Discordance: Are Planck CMB and CFHTLenS weak lensing measurements out of tune? Mon. Not. R. Astron. Soc. 2015, 451, 2877-2888. [CrossRef]

41. Di Valentino, E.; Melchiorri, A.; Silk, J. Beyond six parameters: Extending $\Lambda$ CDM. Phys. Rev. D 2015, 92, 121302. [CrossRef]

42. Di Valentino, E.; Giusarma, E.; Mena, O.; Melchiorri, A.; Silk, J. Cosmological limits on neutrino unknowns versus low redshift priors. Phys. Rev. D 2016, 93, 083527. [CrossRef]

43. Calabrese, E.; Slosar, A.; Melchiorri, A.; Smoot, G.F.; Zahn, O. Cosmic Microwave Weak lensing data as a test for the dark universe. Phys. Rev. D 2008, 77, 123531. [CrossRef] 
44. Di Valentino, E.; Melchiorri, A.; Linder, E.V.; Silk, J. Constraining dark energy dynamics in extended parameter space. Phys. Rev. D 2017, 96, 023523. [CrossRef]

45. Di Valentino, E.; Melchiorri, A.; Silk, J. Reconciling Planck with the local value of H0 in extended parameter space. Phys. Lett. B 2016, 761, 242-246. [CrossRef]

46. Renzi, F.; Di Valentino, E.; Melchiorri, A. Cornering the Planck $A_{l e n s}$ anomaly with future CMB data. Phys. Rev. D 2018, 97, 123534. [CrossRef]

47. Cabass, G.; Di Valentino, E.; Melchiorri, A.; Pajer, E.; Silk, J. Constraints on the running of the running of the scalar tilt from CMB anisotropies and spectral distortions. Phys. Rev. D 2016, 94, 023523. [CrossRef]

48. Ooba, J.; Ratra, B.; Sugiyama, N. Planck 2015 constraints on the non-flat XCDM inflation model. arXiv 2017, arXiv:1707.03452.

49. Muñoz, J.B.; Grin, D.; Dai, L.; Kamionkowski, M.; Kovetz, E.D. Search for Compensated Isocurvature Perturbations with Planck Power Spectra. Phys. Rev. D 2016, 93, 043008. [CrossRef]

50. Valiviita, J. Power Spectra Based Planck Constraints on Compensated Isocurvature, and Forecasts for LiteBIRD and CORE Space Missions. J. Cosmol. Astropart. Phys. 2017, 4, 14. [CrossRef]

51. Capozzi, F.; Di Valentino, E.; Lisi, E.; Marrone, A.; Melchiorri, A.; Palazzo, A. Global constraints on absolute neutrino masses and their ordering. Phys. Rev. D 2017, 95, 096014. [CrossRef]

52. Kilbinger, M.; Heymans, C.; Asgari, M.; Joudaki, S.; Schneider, P.; Simon, P.; Van Waerbeke, L.; Harnois-Déraps, J.; Hildebrandt, H.; Köhlinger, F.; et al. Precision calculations of the cosmic shear power spectrum projection. Mon. Not. R. Astron. Soc. 2017, 472, 2126-2141. [CrossRef]

(C) 2018 by the authors. Licensee MDPI, Basel, Switzerland. This article is an open access article distributed under the terms and conditions of the Creative Commons Attribution (CC BY) license (http:// creativecommons.org/licenses/by/4.0/). 